\title{
Older patients are at increased risk for renal posttransplantation diabetes mellitus
}

\author{
Ana Laura Pimentel, Priscila Aparecida Correa Freitas*, Joíza Lins Camargo \\ From 20th Brazilian Diabetes Society Congress \\ Porto Alegre, Brazil. 11-18 November 2015
}

\section{Background}

Posttransplantation diabetes mellitus (PTDM) is a metabolic complication related to the use of immunosuppressive medication after renal transplant. Identifying patients at highest risk of PTDM would help clinicians in their management, once PTDM is associated with higher number of graft rejection and death in the longterm.

\section{Objectives}

The aim of our study was to determine the risk factors associated with PTDM development at the fourth month after renal transplantation.

\section{Materials and methods}

All patients without diabetes who underwent renal transplant at a University Hospital between July 2012 and June 2015 were included. PTDM was diagnosed according to current ADA criteria at four months after transplantation. Poisson regression with robust standard errors was performed with PTDM as dependent variable and the possible risk factors under study (age, sex, type of donor, immunosuppressive type, family history of DM, pre-transplant BMI and fasting plasma glucose) as independent variables. P-value $<0.05$ was considered as statistically significant.

\section{Results}

One hundred fifty-eight patients were included in the study and $24.1 \%$ had PTDM diagnosed at four months after transplantation $(50.6 \%$ men, mean age $46.1 \pm 13.1$ yrs.). The only factor associated with PTDM in our cohort was age $(\mathrm{p}<0.001$; relative risk 1.064 [1.033$1.095])$. Each one year increase in age was associated with $6.4 \%$ higher risk for PTDM.

\footnotetext{
* Correspondence: bio.priscilaa@gmail.com

IPA, Porto Alegre, Brazil
} 